\title{
EVALUACIÓN DE LA TRANSPARENCIA EN LOS ARCHIVOS HISTÓRICOS PROVINCIALES
}

\author{
Ana Reyes Pacios Lozano* \\ Departamento Biblioteconomía y Documentación. Universidad Carlos III de Madrid. \\ Sara Martínez Cardama** \\ Departamento Biblioteconomía y Documentación. Universidad Carlos III de Madrid. \\ Manuela Moro Cabero*** \\ Departamento Biblioteconomía y Documentación. Universidad de Salamanca.
}

\begin{abstract}
Resumen: Se presentan los resultados de transparencia obtenidos mediante la aplicación TransPa_BA en las páginas web de 52 archivos históricos provinciales españoles, además del Real y General de Navarra. Esta herramienta se inspira y utiliza parámetros de la Metodología de Evaluación y Seguimiento de la Transparencia (MESTA). A partir de esta, se proponen 22 indicadores para la medición de la publicidad activa en los archivos que atienden a los requerimientos de la Ley de Transparencia. Los indicadores y sus correspondientes atributos (contenido, forma, accesibilidad, reutilización, datación y actualización) ofrecen un marco para que los archivos aumenten en transparencia rindiendo cuentas de su actividad y principales resultados a la sociedad y, en particular, a sus grupos de interés. Los resultados obtenidos indican que, aunque hay ejemplos de buenas prácticas, la información relacionada con la transparencia es escasa. Las mejores se relacionan con el cuadro de clasificación, la colaboración y cooperación y las estadísticas. Es abundante la información que figura enmascarada en otras categorías y dispersa en el sitio web. Las mejoras, por tanto, deben realizarse no solo a nivel de publicidad activa sino de organización de la información, ya que ni la visibilidad ni la accesibilidad a esta información son las más idóneas. Se espera que este trabajo promueva un aprendizaje colectivo y contribuya a que se vaya incorporando gradualmente información que refuerce la transparencia activa de estas instituciones documentales o, si fuera el caso, eliminar elementos redundantes.

Palabras clave: Transparencia; publicidad activa; TransPa_BA; archivos históricos provinciales; comunicación institucional; indicadores.
\end{abstract}

Title: ASSESSMENT OF TRANSPARENCY IN PROVINCIAL HISTORIC ARCHIVES.

Abstract: The article discusses the findings on website transparency for 52 Spanish provincial historic archives and the Archivo Real y General de Navarra [royal and general archive of the province of Navarra], delivered by TransPa_BA software. This tool is based on and uses the parameters defined in the Metodología de Evaluación y Seguimiento de la Transparencia [transparency assessment and monitoring method, Spanish initials, MESTA]. A total of 22 indicators are proposed to measure active public disclosure by archives in keeping with the provisions of the national Transparency Act. The indicators and their respective parameters (form, reusability, dating and currency) constitute guidelines to enable archives to enhance transparency and accountability, reporting their activities and performance in key areas to society in general and their stakeholders in particular. Although the findings reveal some good practices, scant transparency-related information is provided. The improvements suggested refer not only to public disclosure, but also to how information is arranged, for neither visibility nor accessibility is optimal. This study will hopefully encourage collective learning, contributing to the gradual inclusion of more transparent information and the exclusion of any redundant elements present on these institutions' websites.

Keywords: Transparency; active public disclosure; TransPa_BA; provincial historic archives; institutional communication; indicators.

Copyright: (C) 2021 Servicio de Publicaciones de la Universidad de Murcia (Spain). Este es un artículo de acceso abierto distribuido bajo los términos de la licencia Creative Commons Reconocimiento 4.0 Internacional (CC BY $4.0)$.

\section{INTRODUCCIÓN}

La transparencia constituye la base y herramienta fundamental de gobiernos abiertos y es determinante para la construcción de democracias más sólidas y participativas. Muy ligada a la rendición de cuentas (accountability) de las instituciones, también es el mecanismo que ampara el derecho al acceso a la información de la ciudadanía. Estas dos perspectivas, el de publicidad activa y pasiva, se tienen en cuenta en la Ley 19/2013, de 9 de diciembre, de Transparencia,

\footnotetext{
*areyes@bib.uc3m.es

**smarti1@bib.uc3m.es

*** moroca@usal.es
}

Recibido: 11-05-2021; $2^{\mathrm{a}}$ versión: 14-06-2021; aceptado: 22-06-2021.

PACIOS LOZANO, A.R.; MARTÍNEZ CARDAMA, S. y MORO CABERO, M. Evaluación de la transparencia en los archivos históricos provinciales. Anales de Documentación, 2021, vol. 24, nº 2. Disponible en: http://dx.doi.org/10.6018/analesdoc.479881. 
Acceso a la Información Pública y Buen Gobierno (en adelante, LTAIPBG) (España, 2013), constituyendo una obligación, la primera y un derecho, la segunda. La publicidad activa debe materializarse a través de la publicación de información sobre las actividades y funciones de instituciones públicas, de una manera clara, estructurada, entendible y también en formatos reutilizables (LTAIPBG, cap. II, art. 5, punto 4) (España, 2013).

Las bibliotecas y archivos públicos dependientes de la Administración se ven, por tanto, afectados por esta Ley de Transparencia, y deben publicar información que muestre el modelo de gestión, la posición ante el servicio ofrecido y el resultado de su actividad y servicios. Desde la perspectiva de los archivos, el debate, no obstante, se centra en la concepción del archivo como "garante de la transparencia", y como su "facilitador". Así, Villoria señala que un buen sistema de archivos es requisito imprescindible para la transparencia y el acceso a la información. Casadesús-de-Mingo y Cerrillo-i-Martínez (2018) consideran los sistemas de gestión documental como un elemento esencial para incrementar la transparencia a lo largo de todo el ciclo de vida de los documentos, siendo determinante para frenar la corrupción en la gestión. Estos autores, urgen además a cambiar la legislación en archivos y gestión documental para acercarlos a la transparencia y la rendición de cuentas. Camacho-Mateos (2014) también incide en la mejora de los sistemas de gestión de documentos a la hora de dar a conocer la trazabilidad de los documentos a lo largo de su ciclo.

Como puede verse, la línea argumental pivota en la consideración del archivo como apoyo para la transparencia externa, para la mejora del acceso a la información, y, en definitiva, de la democracia y la calidad de los sistemas democráticos. No obstante, el valor de la transparencia radica en generar confianza en los ciudadanos respecto a las actividades que realizan las administraciones y gobiernos (Giménez-Chornet, 2017, p. 119), incluidos los archivos.

Otra línea de investigación ha sido la evaluación de las webs de los archivos como herramienta de comunicación institucional y de acceso a la información. Esta siempre ha tenido menor repercusión que los modelos de evaluación realizados sobre otras unidades como bibliotecas, por ejemplo. Se perciben dos perspectivas. La primera una generalista, basada en perspectivas webmétricas y de accesibilidad (Chaín-Navarro y Más-Bleda, 2009) que utilizan criterios generales para la evaluación de sus sedes web, y la segunda, basada en el acceso a los contenidos y productos, que, si bien recoge elementos como la publicidad de cuadros de clasificación, el inventario o los mecanismos de descripción, no los integra dentro de un conjunto de indicadores necesarios para la publicidad activa, sino más bien para la modelización de un sistema de diseño o estructura para las webs de las instituciones archivísticas (Sanz-Caballero y Faba-Pérez, 2012).

Sin embargo, acerca de la transparencia propia del archivo como institución que gestiona recursos y debe mostrar documentos relacionados con su gestión y rendimiento de su servicio, existe todavía poco debate académico y profesional. Estos instrumentos de gestión sobre los que los archivos deben de dar publicidad, los clasificó Sheelings (2006) en tres categorías: 1) los que definen la posición del servicio de archivo en la institución (misión, dependencia organizativa, presupuesto, informe anual, documentos de autoevaluación, cuentas anuales y lista de personal y organigrama); 2) los que son útiles para la elaboración de una política de archivos en un campo bien definido (ej. procedimiento de acceso a los documentos, reglamento, planes de desastres, etc.); y 3) los que son útiles en la elaboración de una política que una las misiones de base de la institución con el archivo (ej. política de gestión de los documentos, plan e informe de participación del servicio de archivo en la política de investigación, de calidad de la institución, etc.). En la misma línea, Borrás (2006) considera que la publicidad de elementos de gestión y evaluación institucional como el Cuadro de Mando Integral son esenciales para el progreso de los archivos, y en este caso para la mejora de su transparencia interna.

Lo mismo sucede con la información económica o estadística, obligación recogida en la LTAIPBG (España, 2013), artículo 8.1, d, y que aplica a cuestiones como el presupuesto de cada centro y su seguimiento y ejecución.

Pérez-Santana (2018) es especialmente crítico con la ausencia de información estadística de los archivos en España. Así, señala que no es posible saber cuestiones como “¿Cuánto cuesta un archivo”? o ¿Cuánto dinero público se invierte en su mantenimiento?" dada la escasa información estadística que se obtiene a nivel nacional. En este sentido se alude a la falta de uniformidad a la hora de definir indicadores y poder intercambiar datos de manera interoperable. Pacios y Martínez-Cardama (2020) recogen buenas prácticas de transparencia económico-financiera internacional en las webs de los National Archives and Records Administration (EEUU), The National Archives (UK), National Archives of Australia y el Archivo General de la Nación de Uruguay. La investigación recoge datos sobre indicadores derivados del artículo 8 sobre información económica de la LTAIPBG como memorias, presupuesto y ejecución, contrataciones y otros. Cabe señalar el vacío de esta información económica en los archivos españoles, algo que sorprende en archivos históricos de renombre internacional como los de Simancas, General de Indias y el de la Corona de Aragón que forman parte del registro de la "Memoria del mundo", de la UNESCO. De facto, para los 10 archivos de titularidad estatal se aportan datos generales y anuales desde el 2003, reunidos bajo estadística básica sobre fondos, personas, infraestructuras 
y recursos, volumen de actividad, disponibilidad de servicios, acciones difusoras y consultas, todo ello procesado en tablas o/y gráficas con presentación para su explotación en formatos diferentes (Ministerio de Cultura y Deporte, 2021).

La investigación sobre publicidad activa en archivos que antecede a la realización de TransPa_BA suscribe esta situación (Pacios y La-Torre, 2018; Pacios y Cerdá, 2019). En concreto destaca para el presente trabajo el antecedente de la obra de Pacios y La Torre (2018) que verifica una serie de indicadores relacionados con la LTAIPBG en archivos históricos españoles. Este antecedente ya señaló la falta de documentación sobre la transparencia interna de una muestra de 61 archivos históricos (8 estatales y 53 provinciales), siendo muy homogéneos en la información ofrecida. Es común, por tanto, que estos archivos faciliten el acceso a documentos como cuadros de clasificación, normativa o cartas de servicio, pero no a otro tipo de datos de interés como gastos, inversiones y documentos de evaluación y gestión como memorias. La aplicación de TransPa_BA permite, no solo valorar la existencia o no de esta documentación, sino ponderar que la que se presente lo haga en términos correctos de atributos como la forma, la accesibilidad, la reutilización, la datación y la actualización.

\section{MATERIAL Y MÉTODO}

El objetivo de este trabajo es la evaluación de la información sobre publicidad activa relacionada con la transparencia de 53 archivos históricos provinciales de titularidad estatal gestionados por las comunidades autónomas y el Real y General de Navarra, dependiente de la Comunidad Foral, incluido porque comparte con los AHP algunas de las funciones más definitorias, como son la custodia de los protocolos notariales y la documentación histórica de las oficinas del Estado en la provincia, esta última con carácter de depósito (Flores-Varela, 2019, p. 5). En la actualidad algunos están integrados en los regionales, como el de Murcia; otros como el del Reino de Galicia, sin la denominación de "provincial”, tiene asignado el papel de Archivo Histórico Provincial de A Coruña.

Para evaluar la información de la publicidad activa relacionada con la transparencia se accedió a cada sitio web al que se llega desde la web del Ministerio de Cultura y Deporte, sección de archivos, enlace titulado "Archivos de titularidad estatal gestionados por las Comunidades Autónomas" (Ministerio de Cultura y Deporte, s.f.). La sede web de la que se partió para el análisis y posterior valoración se puede ver en el Anexo a este trabajo.

La herramienta de evaluación utilizada es TransPa_BA, fruto de la adaptación de la Metodología de Evaluación y Seguimiento de la Transparencia (en adelante, MESTA). Esta evaluación tiene una doble finalidad, en primer lugar mostrar la validez de TransPa_BA a la hora de sistematizar y medir los elementos necesarios de publicidad activa sobre transparencia y, en segundo lugar, tratar de generar debate e interés sobre la necesidad de la puesta a disposición pública de esta información por parte de los archivos, y de que la transparencia sea entendida desde una doble perspectiva: externa, considerando a los archivos herramienta indispensable para su consecución, e interna, como ejemplo de rendición de cuentas de las instituciones hacia sus ciudadanos.

TransPa_BA, es fruto de varias etapas metodológicas consistentes en una investigación previa sobre aplicación de indicadores en bibliotecas y archivos (Pacios, 2003; Pacios y La-Torre, 2018). El grupo de indicadores resultantes se validó y adaptó según MESTA, sistema oficial de cumplimiento de la transparencia para medir la aplicación de la LTAIPBG en los distintos organismos.

Teniendo en cuenta que no todas las instituciones obligadas por la ley tienen la misma capacidad jurídica y normativa, los requerimientos y obligaciones de transparencia no son iguales (Ros-Medina, 2018). Tras eliminar aquellos requisitos que ni un archivo o biblioteca podían cubrir, se fijaron 20 indicadores-marco para la evaluación de unidades documentales, siendo 20 y 21 para bibliotecas (según se trate de públicas o universitarias) y 22 para los archivos. Posteriormente se abrió un proceso de validación de los indicadores a los profesionales en archivos y bibliotecas, mediante una encuesta a los 205 directores de bibliotecas y archivos de todas las tipologías que cubría el proyecto (archivos históricos provinciales, archivos universitarios, bibliotecas públicas del estado y bibliotecas de las universidades públicas) (Pacios, Vianello y De-la-Mano, 2020).

Los 22 indicadores de TransPa_BA para archivos están agrupados en 8 áreas, que son las siguientes: propósito del servicio y objetivos que persigue; órganos de gobierno y reglas de funcionamiento; ofertas de servicio; el fondo documental; personal; resultados; información económica y, finalmente, colaboración/cooperación. La búsqueda de los indicadores en las sedes web de los archivos se realizó en los meses de febrero y marzo de 2021. Cada indicador tiene una ponderación concreta en el resultado total posible que asciende a 100 puntos. Esa ponderación se obtuvo como resultado de la media obtenida en la encuesta que se hizo a los responsables de los archivos históricos y cuya tasa de respuesta obtenida fue del 35,1\% $(n=54)$. En la encuesta se les preguntó, en una escala de 0 a 5 , tanto por la relevancia 
de cada una de las áreas como por la idoneidad de cada uno de los indicadores. Los 22 indicadores, agrupados en las 8 áreas anteriormente indicadas, se muestran en la tabla I con su correspondiente ponderación.

\begin{tabular}{|c|c|c|c|c|}
\hline & \multicolumn{4}{|l|}{ ARCHIVO HISTÓRICO } \\
\hline & \multicolumn{3}{|l|}{ Presentación de la publicidad activa en la sede web del archivo } & \multirow[t]{2}{*}{$0,01 \%$} \\
\hline & INDICADORES DE PUBLICIDAD ACTIVA & 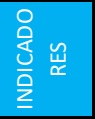 & 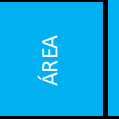 & \\
\hline 1 & \multicolumn{4}{|l|}{ PROPÓSITO DEL SERVICIOY OBJETIVOS QUE PERSIGUE } \\
\hline 1.1 & Definición de la misión & $6,60 \%$ & & \\
\hline \multirow[t]{2}{*}{1.2} & Plan estratégico & $6,16 \%$ & & \\
\hline & TOTAL APARTADO 1 & & $12,76 \%$ & \\
\hline 2 & \multicolumn{4}{|l|}{ ÓRGANOS DE GOBIERNO Y REGLAS DE FUNCIONAMIENTO } \\
\hline 2.1 & Identificación de los miembros que integran la comisión relacionada con la gestión del archivo & $2,76 \%$ & & \\
\hline 2.2 & Reglamento & $3,15 \%$ & & \\
\hline 2.3 & Normativa específica de funcionamiento de los servicios & $3,45 \%$ & & \\
\hline \multirow{2}{*}{2.4} & Código de ética, lista de valores o código de buenas prácticas & $3,32 \%$ & & \\
\hline & TOTAL APARTADO 2 & & $12,68 \%$ & \\
\hline 3 & \multicolumn{4}{|l|}{ OFERTA DE SERVICIOS } \\
\hline \multirow[t]{2}{*}{3.1} & Carta de servicios & $13,31 \%$ & & \\
\hline & TOTAL APARTADO 3 & & $13,31 \%$ & \\
\hline 4 & \multicolumn{4}{|l|}{ EL FONDO DOCUMENTAL } \\
\hline 4.1 & Política / programa de gestión de la colección & $4,56 \%$ & & \\
\hline 4.2 & Cuadro de clasificación y organización de fondos & $4,96 \%$ & & \\
\hline \multirow[t]{2}{*}{4.3} & Calendario de conservación & $3,92 \%$ & & \\
\hline & TOTAL APARTADO 4 & & $13,44 \%$ & \\
\hline 5 & \multicolumn{4}{|l|}{ PERSONAL } \\
\hline 5.1 & Organigrama & $5,98 \%$ & & \\
\hline \multirow[t]{2}{*}{5.2} & Directorio de personal & $5,31 \%$ & & \\
\hline & TOTAL APARTADO 5 & & $11,29 \%$ & \\
\hline 6 & \multicolumn{4}{|l|}{ RESULTADOS } \\
\hline 6.1 & Indicadores de gestión (Cuadro de Mando) & $2,22 \%$ & & \\
\hline 6.2 & Encuestas de satisfacción de usuarios & $2,32 \%$ & & \\
\hline 6.3 & Memoria anual o informe de actividades & $2,88 \%$ & & \\
\hline 6.4 & Reconocimientos, premios, certificaciones & $2,42 \%$ & & \\
\hline \multirow[t]{2}{*}{6.5} & Estadísticas & $2,62 \%$ & & \\
\hline & TOTAL APARTADO 6 & & $12,48 \%$ & \\
\hline 7 & \multicolumn{4}{|l|}{ INFORMACIÓN ECONÓMICA } \\
\hline 7.1 & Presupuesto ejecutado & $4,19 \%$ & & \\
\hline 7.2 & Contratos de licitación y concursos & $3,78 \%$ & & \\
\hline \multirow[t]{2}{*}{7.3} & Subvenciones y ayudas concedidas & $4,07 \%$ & & \\
\hline & TOTAL APARTADO 7 & & $12,04 \%$ & \\
\hline 8 & \multicolumn{4}{|l|}{ COLABORACIÓN / COOPERACIÓN } \\
\hline 8.1 & Redes, grupos de trabajo, comisiones, con las que se trabaja y coopera & $5,96 \%$ & & \\
\hline \multirow[t]{4}{*}{8.2} & Convenios & $6,04 \%$ & & \\
\hline & \multicolumn{2}{|l|}{ TOTAL APARTADO 8} & $12,00 \%$ & \\
\hline & \multicolumn{2}{|l|}{ TOTAL APARTADOS } & $100,00 \%$ & $99,99 \%$ \\
\hline & \multicolumn{3}{|l|}{$\begin{array}{r}\text { TOTAL PUBLICIDAD ACTIVA } \\
\end{array}$} & $100,00 \%$ \\
\hline
\end{tabular}

Tabla I. Ponderación de indicadores de publicidad activa para archivos históricos.

En la publicación de la información relacionada con la transparencia se evalúan los siguientes 6 atributos: contenido, forma, reutilización, accesibilidad, datación y actualización. La escala de valores posibles oscila entre 0 y 10 , a 
excepción de los dos últimos atributos, datación y actualización, cuyos valores oscilan entre 0 y 5 . Cada indicador puede, por tanto, obtener un total máximo de 50 puntos. Además, a la valoración de estos atributos es posible añadir 10 puntos relacionados con la presentación de esta información, en caso de que la sede web del archivo cuente con una sección específica dedicada a la transparencia que reúna esta información. La presentación está referida al lugar de la web en el que se muestra la información y que se traduce en una mayor o menor visibilidad.

En relación con cada uno de los atributos elegidos para valorar los indicadores cabe hacer las siguientes puntualizaciones en algunos:

- Contenido. Aunque no se establecieron contenidos mínimos para cada indicador sí se concretaron ciertos aspectos en relación con la información que debería aparecer en algunos para poder considerar su presencia como tal:

- Misión, debía aparecer con esa denominación, no con otras fórmulas como objetivos o funciones.

- Directorio, solo se consideraron aquellos que contenían algún nombre y forma de contacto.

- Política o programa de gestión de la colección. Se tuvo en cuenta también la política de adquisiciones, aunque se tratara de un documento de entidad inferior por estar relacionado con la gestión del fondo documental.

- Indicadores de gestión, solo se estimaron aquellos que mostraban una continuidad en el tiempo con acceso a resultados, considerando como válidos los referidos a cartas de servicios.

- Redes, grupos de trabajo, comisiones, con las que se trabaja y coopera. Se consideró la presencia de logos o iconos que demostraban la pertenencia a una red con la que colabora el archivo.

- Forma. Dado que no se localizó ningún archivo con portal de transparencia se estimó a efectos de medición de este atributo la página principal del archivo. Hay que indicar que la forma se refiere al lugar y modo de llegar a la información. Directa cuando la información se encuentra en la página principal o mediante link o enlace se sitúa al ciudadano en el contenido de la información, por ejemplo, se cita una normativa, caso del reglamento, y acompaña el enlace a la publicación de esa concreta norma en el BOE y, por tanto, no requiere de una labor de búsqueda. E indirecta, cuando mediante link o enlace se sitúa al ciudadano en la dirección web donde se encuentra la información, pero este debe buscarla.

No se contó con aquellos enlaces desde los que se accede a los diferentes sistemas de archivo o aquellos específicos de estadísticas de archivos que están fuera de la página principal del archivo. Es el caso de enlaces localizados en la web de algunos archivos provinciales con acceso a la página de transparencia o al portal de datos abiertos de la comunidad, pero en los que no se percibe ninguna intención manifiesta por parte del archivo de llegar a ellos. De ahí que el nivel de dificultad de la ciudadanía para localizar las estadísticas concretas de ese archivo sea elevado cuando no imposible.

- Reutilización. Se optó por computar solo dos valores, 0 y 10 y no diferentes niveles dada la variedad de opiniones y ponderaciones existentes en continua evolución desde la propuesta por Berners-Lee (2010) sobre los datos abiertos. El valor 10 se dio a aquellos formatos estructurados que, para su uso, no requerían de la aplicación de técnicas o esfuerzo extra (xls, csv, xml).

Es posible pensar que este atributo no debería ser aplicable a todos los indicadores y que es más propio de aquellos que se muestran en forma de datos como, por ejemplo, las estadísticas. Sin embargo, dado que se desconoce la reutilización que podrían tener estos documentos y teniendo en cuenta los análisis que se vienen realizando de algunos (planes estratégicos, cartas de servicio, memorias, políticas) se decidió tenerlo en cuenta en todos los indicadores.

- Actualización. Se estableció como fecha el año 2019. No obstante, hay que tener en cuenta que no es aplicable a toda la información, ya que no todos los indicadores propuestos requieren de una actualización anual, como por ejemplo una carta de servicios, un reglamento o un plan estratégico. En este último caso, por ejemplo, lo que hubo que verificar es que el plazo que comprende se encuentre en vigor. En cualquier caso, se aplicó el mismo criterio a todos los archivos: si la fecha de actualización correspondía a 2019 se valoró con 10 y, en caso contrario, se calificó con 0 .

Conviene incidir en que TransPa_BA es una herramienta diseñada para que sea utilizada por los responsables o directores de bibliotecas y archivos con interés en la transparencia y en su mejora que se pondrá a su disposición en la siguiente página web: https//www.uc3m.es/investigacion/transpa_ba. Aunque en esta ocasión la evaluación se ha realizado por personas externas y ajenas a la institución, hecho que conlleva hacerlo desconociendo determinada 
información, lo idóneo es que se utilice por personal del archivo. Solo de esta forma se podrán valorar adecuadamente algunos atributos, por ejemplo, la actualización. Es el personal del archivo el que sabe si determinada información requiere o no de actualización y, por tanto, si este atributo es susceptible de valorarse con la máxima puntuación aunque no coincida con el año fijado para la evaluación, algo que una persona ajena desconoce cuando hace la evaluación. Así, pudiera darse el caso de que el resultado final del índice de transparencia sea inferior al real, siendo así infravalorada la situación de transparencia en ese archivo en cuestión.

\section{RESULTADOS Y BUENAS PRÁCTICAS}

\subsection{Resultados}

La aplicación de TransPa BA a los archivos históricos provinciales españoles arroja resultados tanto sobre los indicadores y atributos seleccionados para la medición, como sobre la disposición y organización de la información en las páginas de los archivos. En este sentido, es preciso aludir a la dificultad que supuso localizar la información en las páginas web, ya que esta se encuentra dispersa, y en ningún caso se agrupa bajo un enlace común. Bien es cierto que algunos de los indicadores se agrupan, aunque en pocos archivos, bajo enlaces titulados como "Información general" y "Quiénes somos".

La búsqueda de los diferentes indicadores a través de las sedes web de los archivos permitió identificar aspectos que demuestran un mayor interés por mostrar y dar acceso a información relacionada con la transparencia en unos archivos frente a otros, demostrándose así que la transparencia es también una cuestión de voluntad. Así, por ejemplo, suele ser habitual que los archivos de una comunidad autónoma compartan el mismo modelo de sede web. Es aquí donde, a pesar de contar con común diseño y estructura, se perciben las diferencias y revelan que existe un margen de actuación e influencia de los responsables del archivo en los contenidos dispuestos para los usuarios. Uno de los ejemplos más notables reside en las estadísticas, en las que se observaron variantes en la forma de acceso y también en los formatos disponibles. Un aspecto positivo es la posibilidad de compartir documentos comunes por todos ellos, como por ejemplo las reglas y calendarios de conservación y el cuadro de clasificación de los archivos provinciales de la Comunidad de Castilla y León.

En lo que concierne a los atributos considerados (presentación, contenido, forma, reutilización, datación y actualización), cabe hacer las siguientes observaciones, a excepción de la accesibilidad a los indicadores que es buena en el $96,07 \%$ de los casos:

\subsubsection{Sobre la presentación}

No se localizó ningún archivo que disponga de un enlace, sitio o espacio titulado y dedicado a la transparencia. Ninguno de los indicadores parece asociarse con la misma.

\subsubsection{Sobre el contenido}

En relación con los indicadores que aparecen en el conjunto de los 53 archivos, la tabla II muestra los localizados. De un total de 22 se localizaron 16. La suma de documentos asciende a 229. Se presentan ordenados de mayor a menor presencia con el número del epígrafe correspondiente al área de TransPa_BA. En la columna de la derecha aparece el porcentaje que supone el indicador sobre los 53 archivos analizados. 


\begin{tabular}{|c|c|c|}
\hline INDICADORES & TOTAL & $\%$ \\
\hline 4.2 Cuadro de clasificación y organización de fondos & 49 & $92.45 \%$ \\
\hline 8.1 Redes, grupos de trabajo, comisiones, con las que se trabaja y coopera & 35 & $66.04 \%$ \\
\hline 6.5 Estadísticas & 34 & $64.15 \%$ \\
\hline 1.1 Definición de la misión & 23 & $43.40 \%$ \\
\hline 2.3 Normativa específica de funcionamiento de los servicios & 20 & $37.74 \%$ \\
\hline 3.1 Carta de servicios & 19 & $35.85 \%$ \\
\hline 5.2 Directorio de personal & 11 & $20.75 \%$ \\
\hline 6.3 Memoria anual o informe de actividades & 10 & $18.87 \%$ \\
\hline 4.3 Calendario de conservación & 9 & $16.98 \%$ \\
\hline 6.1 Indicadores de gestión (Cuadro de mando) & 6 & $11.32 \%$ \\
\hline 6.2 Encuestas de satisfacción de usuarios & 5 & $9.43 \%$ \\
\hline 4.1 Política / programa de gestión de la colección & 4 & $7.55 \%$ \\
\hline 2.2 Reglamento & 1 & $1.89 \%$ \\
\hline 2.4 Código de ética, lista de valores o código de buenas prácticas & 1 & $1.89 \%$ \\
\hline 5.1 Organigrama & 1 & $1.89 \%$ \\
\hline 7.1 Presupuesto ejecutado & 1 & $1.89 \%$ \\
\hline
\end{tabular}

Tabla II. Indicadores localizados.

Los resultados son coherentes con los presentados en el trabajo de Pacios y La-Torre (2018), en el que analizaron 18 indicadores. Los porcentajes ofrecidos en el análisis de archivos históricos provinciales son similares, si bien algo superiores en el estudio de 2021. Elementos como la misión, normativa específica o cartas de servicios alcanzan similares porcentajes, mientras que otros, como el organigrama o el presupuesto, siguen siendo elementos residuales en estas páginas web.

El Cuadro de clasificación es el que tiene la mayor presencia en las webs con $92,45 \%$ (frente al $88,7 \%$ del estudio de 2018). Le siguen las redes o grupos de colaboración con un $66,04 \%$ algo que sí ha mejorado sustancialmente en los logotipos de las webs. Las estadísticas, que en el estudio de 2018 no se contemplaban como tal, sí destacan como uno de los indicadores más frecuentes. La definición de la misión también incrementa su frecuencia, por ejemplo, de un $22,6 \%$ en 2018 a un $43,40 \%$. Acontece idéntico proceder alcista con indicadores como la normativa de funcionamiento de servicios. Por último, nos encontramos con indicadores que mantienen el valor como es el caso de la carta de servicios o el directorio.

\subsubsection{Sobre la forma}

Se percibe un ligero incremento a la hora de acceder a la información de manera directa (130) frente al modo indirecto (99). Es decir, los archivos muestran más información relacionada con los indicadores de forma directa que indirecta. Por ejemplo, a la hora de citar una normativa o reglamento se acompaña directamente a su enlace y publicación. En otros casos, se hace de forma indirecta, como cuando el archivo enlaza a la página de la consejería en la que se encuentran las estadísticas y el usuario debe buscar las estadísticas de ese archivo en particular.

Se dan situaciones en las que la localización de la información está particularmente desubicada en relación con el archivo afectado y un ciudadano tendría muchas dificultades para llegar a ella. Son ejemplo aquellos archivos que disponen de un mismo modelo de banner con información compartida para todos los archivos de la comunidad en donde se ubica el acceso a las estadísticas. Así, por ejemplo, en los archivos de la Comunidad de Madrid se puede acceder a una sección denominada "Estadística de archivos" en la que se presentan, desde 2012 y con periodicidad bienal, hasta el 2018, las tablas de resultados clasificadas en 14 categorías de datos de interés archivístico: Características generales; edificios de archivos; capacidad de almacenamiento; accesibilidad; servicios y equipamientos; informatización; Sistemas de gestión de documentos; Usuarios; Actividades educativas y culturales; Personal; Presupuestos; externalización de servicios; carácter y volumen de los fondos y restauración. Además, en los últimos dos bienios con formatos reutilizables (xls). Sin embargo, el AH de Protocolos, objetivo de este trabajo, no utiliza ni enlaza a estos datos en su sección titulada "El archivo en cifras" para ampliar la información que presenta, dentro de su propia sede web, en la que no aparece, por ejemplo, dato alguno relacionado con los presupuestos. 
En esta misma línea, algunos archivos disponen de un enlace que permite llegar al portal de la transparencia de la comunidad a la que pertenecen en donde su información no es fácil de localizar al estar dispersa entre todo tipo de datos, y con suerte, unidos a los de otras entidades culturales como bibliotecas y museos.

\subsubsection{Sobre la reutilización}

En relación con este atributo hay que advertir la escasa presencia de formatos reutilizables en entidades como estas que se dedican a gestionar la información. La LTAIPBG (España, 2013) indica en su cap. II, art. 5.4: "la información sujeta a las obligaciones de transparencia será publicada en las correspondientes sedes electrónicas o páginas web y de una manera clara, estructurada y entendible para los interesados y, preferiblemente, en formatos reutilizables".

Los formatos más comunes son, por orden de frecuencia, pdf y html. De los 229 documentos o información relacionada con los indicadores, solo 11 corresponden a formatos reutilizables. En formato xls y csv están accesibles los datos estadísticos que ofrecen los archivos de Castilla y León. En el banner superior de su web todos presentan un enlace titulado "Actividades y recursos" que da acceso a otro denominado "Datos abiertos de archivos". En este último, se pueden encontrar para su descarga los datos básicos de todos los archivos de la comunidad, en los formatos antecitados xls y csv. Asimismo, desde la web de los AHP de la Comunidad de Andalucía, a través del enlace "El archivo en cifras" se llega a las estadísticas de archivos de la Consejería de Cultura y Patrimonio Histórico, en formato xls y pdf. Solo los de Málaga y el de la Chancillería de Granada no siguen esta práctica, como ya se comentó a propósito de los modelos comunes de sedes web localizados, pero con diferencias en algunos aspectos como las estadísticas.

Otra excepción en cuanto a formatos reutilizables se observó en el cuadro de clasificación y organización de fondos que, solo en el caso de los archivos de Zaragoza y Huesca, conduce a un enlace al Sistema de Información de Archivos de Aragón DARA, página con información en formato xml.

\subsubsection{Sobre la datación}

Cualquier información o documento publicado debería disponer de una fecha de datación con el fin de situar al ciudadano en el tiempo. Más aún cuando se trata de documentación que, como un cuadro de clasificación, constituye una herramienta fundamental en los archivos al servir de base para realizar algunas de las tareas archivísticas e informar al usuario sobre los fondos, además de contribuir a facilitar su búsqueda. Del total de los 229 indicadores localizados, 122 estaban datados $(53,28 \%)$, siendo las estadísticas y el cuadro de clasificación los más comunes.

\subsubsection{Sobre la actualización}

Por lo que se refiere a este atributo, que indica la revisión de la información en la fecha elegida (2019), 56 documentos de los 229 estaban actualizados (24,45\%), ocupando las estadísticas el primer lugar. Hay que considerar aquí lo ya indicado en relación a que no todos los documentos e información requieren de una actualización anual.

\subsection{7 Índice de transparencia en publicidad activa}

Aunque el objetivo de este trabajo no es hacer un ranking sino contribuir a avanzar en la transparencia de estas instituciones, como ya se indicó anteriormente, la aplicación de la herramienta TransPa_BA lleva a la obtención de un cómputo final en publicidad activa para cada archivo lo que permite posicionarlos. La correspondiente evaluación de los atributos establecidos para cada indicador posibilita obtener unos resultados totales en los archivos que se presentan en la tabla III. Los valores asociados al índice de transparencia obtenido para cada archivo son bajos. Se debe, esencialmente, al valor 0 de algunos atributos como la reutilización, datación o la actualización. Hay que tener en cuenta la situación a la que se aludió ya en relación con este último, la posibilidad de realizar una valoración errónea si un determinado indicador no requiere de una actualización. Es necesario por ello tomar los índices de transparencia que se presentan como un valor aproximado que sirva para orientar hacia la mejora de los atributos que debería tener la información relacionada con los indicadores.

Los resultados muestran que el Archivo Histórico Provincial de Murcia, integrado en el regional, ocupa la primera posición, con 31,19 puntos y 9 indicadores, seguido de los archivos provinciales de Almería y Granada con 27,09 y 26,76, respectivamente, ambos con 7 indicadores. Hay tres archivos en los que no se localizó información alguna relacionada con la publicidad activa y 21 no llegan a 10 puntos sobre 100, lo que vislumbra la necesidad de mejorar la transparencia en estos archivos.

Los diez archivos que se muestran más transparentes son los de las provincias de Murcia, Almería, Granada, Cádiz, Sevilla, Córdoba, Jaén, Madrid, Burgos y León. Con puntuaciones desde 31,19 (Murcia) a 23,95 (León). Ello pese al déficit evidente de indicadores presentes en sus sedes web, dado que los citados tienen entre 9 y 6 indicadores de los 22 propuestos. 
La imagen que proporciona la tabla III facilita que cada archivo pueda conocer su situación en materia de transparencia y, en consecuencia, decidir sobre su mejora trabajando para hacer accesible aquella información que se corresponde con los indicadores ausentes, considerando además el cumplimiento de los atributos que debería tener si se pretende seguir la ley.

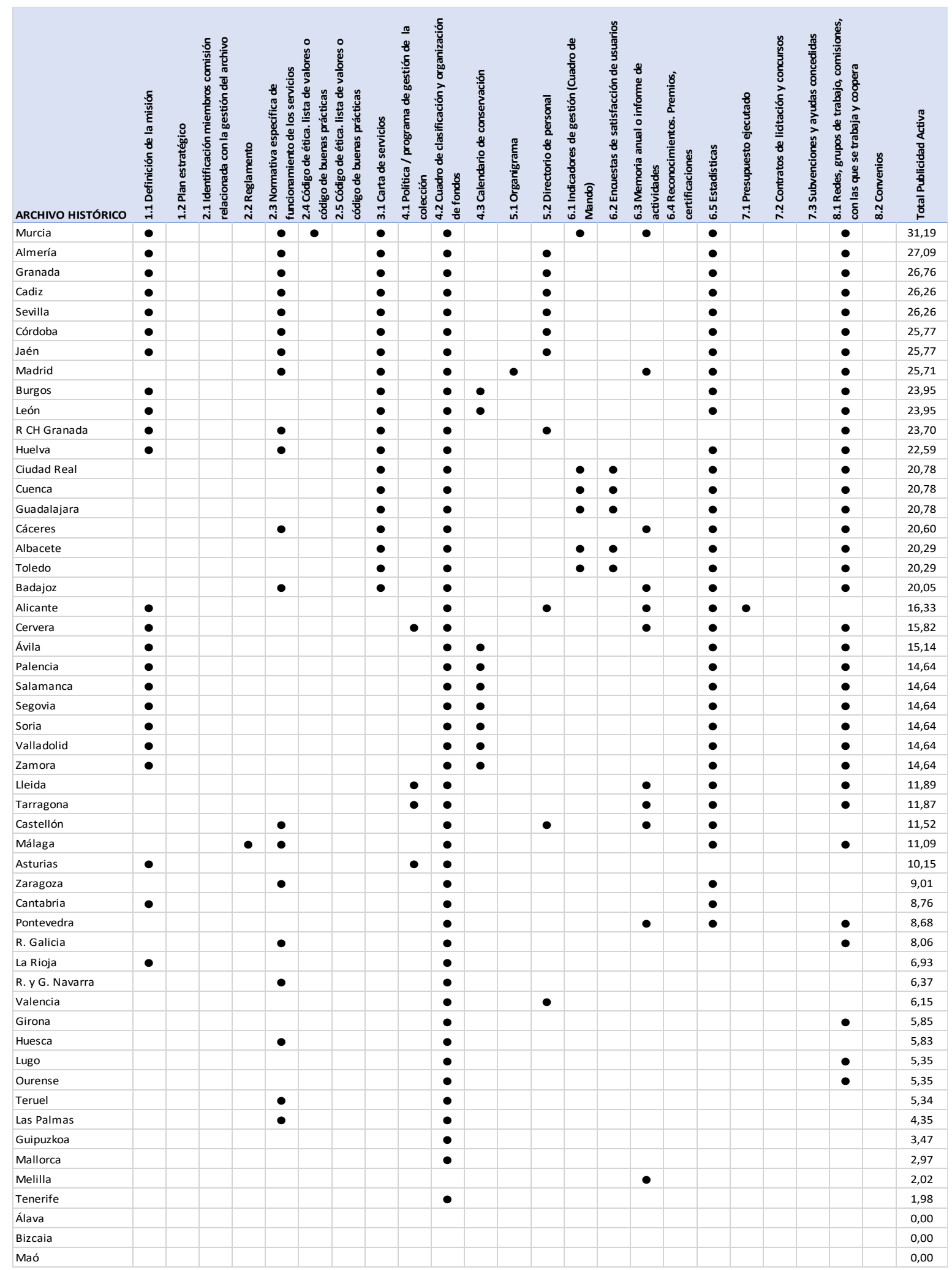

Tabla III. Índice de transparencia en publicidad activa en los archivos históricos provinciales. 


\subsection{Buenas prácticas en transparencia en los archivos históricos provinciales}

La búsqueda y valoración de los indicadores de transparencia mediante la herramienta tecnológica TransPa_BA ha permitido identificar buenas prácticas en la información relacionada con la publicidad activa en algunos de estos archivos y mostrar deficiencias en otros para su corrección y mejora. A continuación, se muestran algunos ejemplos de buenas prácticas:

Cuadro de clasificación. Siendo este el documento más común, instrumento de referencia y control que permite la identificación, la agrupación física y la recuperación de los fondos, cabe señalar diferencias notorias. Aunque la mayoría se han localizado como documentos con enlace propio en la página principal, también los hay que forman parte de otros documentos como la memoria anual y la guía del archivo. Dado su interés consideramos que debe estar bien visible y en sitio o con enlace propio, es decir con acceso directo. Cabe destacar como ejemplos de buenas prácticas, entre otros, los casos de Zaragoza, Huesca con enlace a los fondos de DARA, (Iranzo-Muñío, 2018, p. 84); los de Castilla y León, cuya navegación por el cuadro permite el acceso a la información sobre el fondo o fondos que conserva, descendiendo por los distintos niveles organizativos hasta llegar a la descripción normalizada ISAD del fondo documental; igualmente, señalamos el de Murcia que desciende hasta el nivel de serie y, en algunos casos, hasta expedientes concretos a pesar de tener buscador en la página principal.

Carta de servicios. Se trata de un documento referente a los compromisos de calidad en los servicios prestados, creado para fomentar la transparencia en la Administración Pública (España, 2005) y considerado como una de las principales manifestaciones públicas de transparencia respecto al rendimiento, eficiencia y eficacia de los servicios públicos (Löffler; Parrado y Zmeskal, 2007, p. 18). El del archivo de Murcia representa un buen ejemplo por estar actualizado incluyendo el grado de cumplimiento de los compromisos anuales, actualizado a data de 2020. Indicadores ligados a los compromisos de las cartas de servicio, en su seguimiento anual, especificando así mismo el estándar, se observan en los archivos de Castilla-La Mancha.

Memorias. La memoria es uno de los documentos más completos en la medida que registra estadísticas vinculadas a la unidad informativa, todo tipo de actividades y datos relacionados con los servicios prestados del archivo a modo de rendición de cuentas anual e incluye, además, con frecuencia, relación de nuevas necesidades para sus planes de actuación. A pesar de no ser muy frecuente su observación en este estudio, se localizaron ejemplos que sirven de modelo. Son los casos del archivo de Murcia, el más completo con datos estadísticos, de cooperación, de formación del personal, etc., incluso la relación de los nombres de los profesionales que trabajan en el archivo. Así mismo, las memorias de los archivos de Alicante, Lleida y Tarragona se pueden tomar como referentes.

Directorio. Como guía que permite la localización y contacto de los profesionales que trabajan en el archivo, debería ser una información común en todos. No obstante, su ausencia ha sido en esta investigación una constante. Los localizados son parcos en direcciones de correos electrónicos o teléfono. Muchos se limitan a proporcionar únicamente el contacto del director/a del archivo. Un ejemplo de lo que constituye un directorio completo es el de Alicante, con relación de nombres, categoría profesional y correos electrónicos de contacto de cada uno.

Organigrama. Se refiere a la representación gráfica de la estructura del archivo que incluye los departamentos con las personas que forman parte, mostrando las relaciones jerárquicas y competenciales en vigor, según el grado de detalle al que desciendan. A propósito del mismo la LTAIPBG indica “... incluirán un organigrama actualizado que identifique a los responsables de los diferentes órganos y su perfil y trayectoria profesional" (art. 6.1). Bajo esta premisa solo se localizó uno, el del Histórico de Protocolos de Madrid.

Estadísticas. A pesar de haber considerado como tal a conjuntos de datos muy variopintos que presentan los archivos, es obligado reconocer como buena práctica el enlace que presentan algunos titulados "El archivo en cifras" porque ayuda a obtener una información rápida y práctica sobre contenidos relevantes. Es el caso de los de la Comunidad de Andalucía que, aunque comparten el mismo modelo de página web, hay alguna diferencia entre ellos en lo que se refiere a las estadísticas. La mayoría presentan este enlace así titulado (Almería, Cádiz, Córdoba, Granada, Huelva, Jaén y Sevilla). Dicho enlace provee acceso a la estadística de archivos de Andalucía en la que se pueden localizar las del correspondiente archivo, además en formatos reutilizables. Esos archivos también incluyen un enlace con un documento en pdf con sus datos más actualizados. Málaga utiliza otra denominación en el enlace: "Identificación y datos estadísticos" y proporciona acceso a las estadísticas, pero en formato pdf. En el de la Real Chancillería de Granada no se localizó enlace alguno dedicado a datos estadísticos. También los archivos de Castilla y León, con modelo de web común, presentan un enlace titulado "Datos abiertos de archivos" en el que se accede a datos estadísticos en formatos reutilizables (csv y xls) a través del que se ofrecen los datos del Directorio de Archivos de Castilla y León, el Registro de eliminación de documentos y el Registro de infraestructuras culturales (Figura 1). 


\section{DATOS ABIERTOS DE ARCHIVOS}

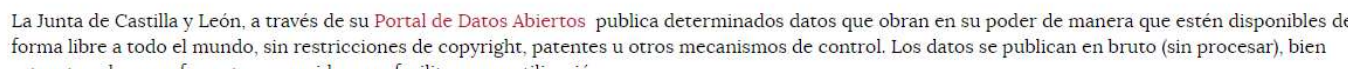

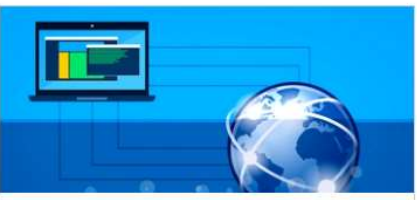

Directorio de Archivos

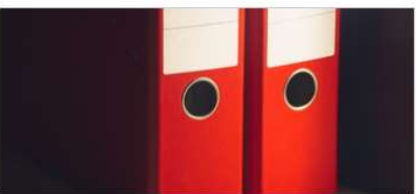

Datos básicos de los Archivos Históricos Provinciales y el

Archivo General de Castilla y

León

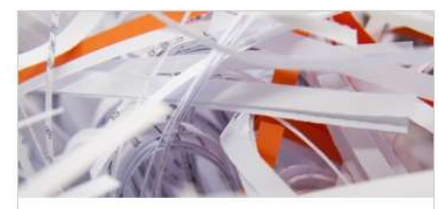

Registro de eliminación de documentos

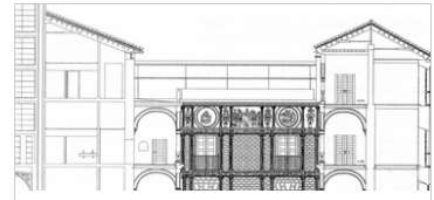

Registro de infraestructuras culturales

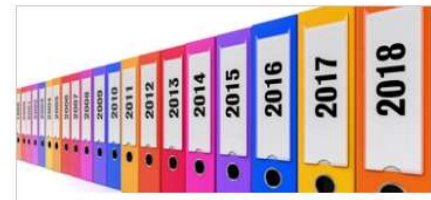

Registro de Reglas de Conservación publicadas
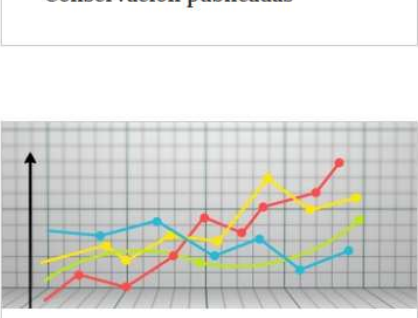

Buscador de conjunto de datos abiertos

Figura 1. Datos abiertos de Archivos de JCYL. Fuente:<https://archivoscastillayleon.jcyl.es/web/es/actividadesrecursos/datos-abiertos-archivos.html>.

Políticas. Aunque no se localizó ninguna política o programa de gestión completa, con todos los procesos que implica, los archivos de Lleida y Tarragona presentan en sus webs una política de adquisiciones que, como indica el propio documento, "aporta principios y criterios que facilitan una distribución ordenada, sistemática y coherente del patrimonio documental y de los documentos del territorio que le es propio, especialmente cuando su titularidad sea pública".

Planes. Pese a haber considerado el plan estratégico como indicador de interés para conocer la estrategia de los archivos, con sus objetivos, actividades y medios empleados para su consecución, y por estar contemplado en la LTAIPBG (art. 6.1) (España, 2013) no se localizó ninguno. Sin embargo, hay que destacar el Plan de actuación de Zaragoza correspondiente al año 2020.

Encuestas de medición de los servicios y, por tanto, reveladoras de la satisfacción de los usuarios se localizaron en todos los archivos de Castilla-La Mancha.

\section{CONCLUSIONES}

La transparencia y rendición de cuentas es una exigencia de las sociedades actuales, no solo hacia sus mandatarios y cargos políticos, sino que, también, hacia el conjunto de instituciones públicas. No en vano, constituye el objetivo 16 de los Objetivos de Desarrollo de la Agenda 2030, por lo que esta rendición de cuentas debe ser transversal a todas las capas de la sociedad.

Los archivos como instituciones públicas se ven obligados por la LTAIPBG a trabajar facilitando y siendo ejemplo de transparencia, no solo desde una perspectiva externa, constituyendo una herramienta para el acceso a la información de otras instituciones, sino también internamente, como institución pública que debe rendir cuentas a la ciudadanía aportando visibilidad a una información que no solo incrementará su transparencia en cuanto a publicidad activa, sino que servirá para mejorar la visibilidad de sus actividades y servicios.

El trabajo que se presenta arroja resultados sobre los indicadores y atributos medidos, sobre la propia herramienta metodológica TransPa_BA y sobre el estado y organización de las webs de archivos en España. Infelizmente, estas carecen, en general, de una buena organización de contenidos resultando complejo sistematizar y evaluar la información 
sobre transparencia ya que, no existe un apartado específico sobre ella en ninguna web y, sobre todo, se observa que es abundante la información que figura enmascarada en otras categorías y dispersa en el sitio web. Las mejoras, por tanto, deben realizarse no solo a nivel de publicidad activa sino de organización de la información, ya que ni la visibilidad ni la accesibilidad son las más idóneas.

En cuanto a la transparencia, medida a través de los indicadores seleccionados y sus atributos, se destaca la ausencia de una implantación significativa de los mismos. El déficit de indicadores es notorio en la mayoría de los sitios analizados. Prueba de ello, es el resultado observado de cumplimiento de 9 indicadores (Murcia) sobre los 22 propuestos.

Los indicadores que figuran al menos en la mitad de los archivos investigados son el cuadro de clasificación, las redes y la información relacionada con la cooperación y las estadísticas. En un rango inferior de cumplimiento se sitúan elementos como la misión, normativas específicas de funcionamiento o las cartas de servicio. Los menos representados, con solo un archivo por cada indicador, son el reglamento (AHP Málaga, aunque del Sistema Andaluz), la relación de valores (AHP Murcia), el organigrama (AH Protocolos de Madrid) o el presupuesto ejecutado (AHP Alicante).

La información sobre sus objetivos y su evaluación es escasa, lo cual dificulta el conocimiento de prioridades de los archivos histórico provinciales, a qué dedican sus recursos o como miden sus resultados. Las limitadas muestras encontradas se convierten en buenas prácticas y modelos a seguir para la mejora e implementación de la transparencia en sus sedes webs.

La herramienta TransPa_BA -en calidad de tecnología cívica- permite medir el nivel de transparencia de publicidad activa en las webs, pudiendo ser eficaz para la mejora interna a la hora de actualizar y plantear nuevos diseños de contenidos en la web. Su aplicación en estos archivos también facilitará observar si los indicadores con sus atributos son los más adecuados para medir la publicidad activa. En cualquier caso, se pretende que esta aplicación genere un efecto positivo y contribuya a mejorar la transparencia de las unidades de información. En este sentido, coincidimos con Giménez-Chornet (2017, p. 113) cuando afirma que "alcanzar la transparencia en la democracia pasa por realizar acciones concretas para fomentar que los ciudadanos ejerzan el control de las instituciones u organismos públicos", incluidos los archivos históricos provinciales.

\section{Agradecimientos}

Trabajo financiado por el Ministerio de Ciencia, Innovación y Universidades, Proyecto RTI2018-095187-B-I00.

\section{BIBLIOGRAFÍA}

BERNERS-LEE, T. Linked Data. 2006 [en línea]. Disponible en: < https://www.w3.org/DesignIssues/LinkedData.html> [Consulta: 25 de abril de 2021]

BORRÁS-GÓMEZ, J. El archivo de la Universidad Pompeu Fabra: valoración del sistema y perspectivas de futuro. En MORENO, A. (ed.) Archivos universitarios. Realidades y proyectos. Valladolid: Universidad de Valladolid, 2006, p. $130-163$.

CAMACHO-MATEOS, B. Los archivos y el acceso a la información pública en España: elementos clave de la transparencia política [en línea]. Salamanca, ArchivPost, 2014. Asociación de Archiveros de Castilla y León. Disponible en: <http://www.acal.es/index.php/archivpost-a-fondo > [Consulta: 25 de abril de 2021]

CASADESÚS-DE-MINGO, A. y CERRILLO-I-MARTÍNEZ, A. Improving records management to promote transparency and prevent corruption. International Journal of Information Management, 2018, vol. 38, $\mathrm{n}^{\mathrm{o}} 11$, p. 256261. Disponible en: https://doi.org/10.1016/j.ijinfomgt.2017.09.005.

CHAÍN-NAVARRO, C. y MÁS-BLEDA, A.M. Los archivos nacionales hispanoamericanos y la gestión de sus websites. Biblios: Revista electrónica de bibliotecología, archivología y museología, 2009, vol. 35, nº 2.

ESPAÑA. Ley 19/2013, de 9 de diciembre, de Transparencia, Acceso a la Información Pública y Buen Gobierno [en línea] 2013. Boletín Oficial del Estado, 10 de diciembre de 2013, núm. 295. Disponible en: $<$ https://www.boe.es/buscar/pdf/2013/BOE-A-2013-12887-consolidado.pdf> [Consulta: 25 de abril de 2021]

ESPAÑA. Real Decreto 951/2005, de 29 de julio, por el que se establece el marco general para la mejora de la calidad en la Administración General del Estado. Boletín Oficial del Estado, de 11 de septiembre de 2005, núm. 211. Disponible en: <https://www.boe.es/diario_boe/txt.php?id=BOE-A-2005-14836> [Consulta: 25 de abril de 2021]

FLORES-VARELA, C. ¿Para qué sirven los archivos históricos provinciales? Os Sistemas de Arquivo no Século XXI: Profesionais e Institucións nos Tempos Líquido. II Encontró Olga Gallego De Arquivos, 22·11·2019. $<$ https://eprints.ucm.es/id/eprint/60390/1/ParaQueSirvenLos\%20AHP.pdf $>$

GIMÉNEZ-CHORNET, V. La auditoría en la gestión de la transparencia del sector público. Métodos de Información, 2017, vol. 8, no 15, p. 111-123. Disponible en: http://dx.doi.org/10.5557/IIMEI8-N15-111123. 
IRANZO-MUÑIO, M.T. Los archivos históricos en la encrucijada. Archivos públicos y memoria social en Aragón, 2008-2018. Tabula, 2018, no 21, p. 81-105.

LÖFFLER, E.; PARRADO, S. y ZMESKAL, T. Improving Customer Orientation through Service Charters: A Handbook for Improving Quality of Public Services. 2007, [en línea] OCDE, Czech Ministry of Interior. Disponible en: <http://www.sigmaweb.org/publications/38370028.pdf $>$ [Consulta: 25 de abril de 2021]

MINISTERIO DE CULTURA Y DEPORTE. Gestión de los archivos en las comunidades autónomas. S.F. [en línea] Disponible en: <https://www.culturaydeporte.gob.es/cultura/archivos/informacion-general/gestionautonomica.html $>$ [Consulta: 1 de febrero de 2021]

MINISTERIO DE CULTURA Y DEPORTE. Estadísticas de archivos estatales. 2021. [en línea] Disponible en: $<$ http://estadisticas.mecd.gob.es/CulturaDynPx/culturabase/index.htm?type=pcaxis\&path=/t15/p15/a2005/\&file=p caxis> [Consulta: 28 de abril de 2021]

PACIOS, A.R. y LA-TORRE, J.L. Spanish historic archives' use of websites as a management transparency vehicle. Archival Science, 2018, vol. 18, no 2, p. 185-205.

PACIOS, A.R. Management-related information on Spanish university library web pages. The electronic library. 2003, vol. 21, no 6, p. 528-537. Disponible en: https://doi.org/10.1108/02640470310509081.

PACIOS, A.R. y CERDÁ, J. Transparencia en la gestión del archivo como unidad organizativa. Una propuesta de indicadores para su evaluación. XIII Congreso de Archivología del MERCOSUR (15 a 21 octubre, Montevideo) 2019 (en prensa).

PACIOS, A.R.; TORREIRO-RODRÍGUEZ, I. y MORO-CABERO, M. Comunicar transparencia. El caso de los archivos universitarios españoles. Revista General de Información y Documentación, 2019, vol. 29, nº 2, p. 527-551. Disponible en: https://doi.org/10.5209/rgid.66980.

PACIOS, A.R y MARTÍNEZ-CARDAMA, S. Active disclosure of Spanish historic archives' economic-financial information, Archives and Records, 2020. [en línea] Disponible en: https://doi.org/10.1080/23257962.2020.1804342.

PACIOS, A.R.; VIANELLO, M y DE-LA-MANO, M. TransPa_BA, una herramienta para la mejora de la publicidad activa en bibliotecas y archivos. Ibersid: revista de sistemas de información y documentación, 2020, vol. 14, n 22 , p. 55-76.

PÉREZ-SANTANA, F. La estadística de archivos en el marco de la transparencia. Radiografía de 2017. En DIAZ, A. y SIERRA-RODRÍGUEZ, J. (eds). La función de archivo y la transparencia en España. Madrid: Universidad Complutense de Madrid, 2018, p. 99-124.

ROS-MEDINA, J.L. La evaluación de la transparencia en España a debate metodológico: Mesta e índices de transparencia internacional. Revista internacional de transparencia e integridad, 2018, $\mathrm{n}^{\circ} 6$.

SANZ-CABALLERO, I. y FABA-PÉREZ, C. Diseño de un modelo basado en criterios e indicadores de características para la evaluación de los sitios webs de archivos. Revista General de Información y Documentación, 2012, vol. 22, p. 307-331. Disponible en: https://doi.org/10.5209/rev_RGID.2012.v22.39660.

SCHEELINGS, F. Pouvoirs et priorites de l'archiviste universitaire: de la theorie archivistique a la pratique. En MORENO, A. (ed.). Archivos universitarios. Realidades y proyectos. Valladolid: Universidad de Valladolid, 2006, p. 89-110.

SUBDIRECCIÓN GENERAL DE ARCHIVOS Y GESTIÓN DOCUMENTAL DE LA COMUNIDAD AUTÓNOMA DE MADRID. Estadística de archivos de la Comunidad de Madrid. 2018. [en línea]. Disponible en: $<$ http://www.madrid.org/archivos/index.php/servicios/censo-y-estadistica-de-archivos/estadistica-de-archivos> [Consulta: 28 de abril de 2021] 
ANEXO

Relación de archivos objeto de análisis con la URL de su sede web.

\begin{tabular}{|c|c|}
\hline Archivos Históricos & URL web \\
\hline Álava & https://gida.irekia.euskadi.eus/es/entities/1522-archivo-historico-provincial-alava \\
\hline Alicante & http://ceice.gva.es/es/web/archivo-historico-provincial-alicante \\
\hline Albacete & https://cultura.castillalamancha.es/archivos/archivos-historicos-provinciales/archivo-historico-provincial-de-albacete \\
\hline Almería & http://www.juntadeandalucia.es/cultura/archivos/web_es/ahpalmeria \\
\hline Asturias & https://archivosdeasturias.info/feaa/action/portada?buttons $\% 5 b 0 \% 5 d=n e w$ \\
\hline Ávila & https://archivoscastillayleon.jcyl.es/web/es/nuestros-archivos/archivo-historico-provincial-avila.html \\
\hline Badajoz & $\begin{array}{l}\text { http://archivosextremadura.gobex.es/WAREX/live/SistemaArchivistico/JuntaExtremaduraSA/ArchivosHistoricoProvinciale } \\
\text { sSA/ArchivosHistoricoProvincialesBA.html }\end{array}$ \\
\hline Bizcaia & https://gida.irekia.euskadi.eus/es/entities/1523-archivo-historico-provincial-bizkaia \\
\hline Burgos & https://archivoscastillayleon.jcyl.es/web/jcyl/ArchivosCastillaYLeon/es/Plantilla66y33_100/1284346752633/_/__ \\
\hline Cáceres & $\begin{array}{l}\text { http://archivosextremadura.gobex.es/WAREX/live/SistemaArchivistico/JuntaExtremaduraSA/ArchivosHistoricoProvinciale } \\
\text { sSA/ArchivosHistoricoProvincialesCC.html }\end{array}$ \\
\hline Cádiz & http://www.juntadeandalucia.es/cultura/archivos/ahpcadiz \\
\hline Cantabria & https://www.culturadecantabria.com/es/archivo-historico \\
\hline Castellón & http://ceice.gva.es/es/web/archivo-historico-provincial-de-castellon \\
\hline Cervera & https://xac.gencat.cat/ca/llista_arxius_comarcals/segarra/diposit_arxius_cervera/ \\
\hline Ciudad Real & https://cultura.castillalamancha.es/archivos/archivos-historicos-provinciales/archivo-historico-provincial-de-ciudad-real \\
\hline Córdoba & http://www.juntadeandalucia.es/cultura/archivos/ahpcordoba \\
\hline Cuenca & https://cultura.castillalamancha.es/archivos/archivos-historicos-provinciales/archivo-historico-provincial-de-cuenca \\
\hline Girona & https://xac.gencat.cat/ca/Ilista_arxius_comarcals/girones/ \\
\hline Granada & $\begin{array}{l}\text { http://www.juntadeandalucia.es/cultura/archivos/web/contenido?id=0754eda0-58a4-11dd-b44b- } \\
\text { 31450f5b9dd5\&idArchivo=0754eda0-58a4-11dd-b44b-31450f5b9dd5 }\end{array}$ \\
\hline Guadalajara & https://cultura.castillalamancha.es/archivos/archivos-historicos-provinciales/archivo-historico-provincial-de-guadalajara \\
\hline Guipuzkoa & http://oinati.gipuzkoakultura.eus/ \\
\hline Huelva & http://www.juntadeandalucia.es/cultura/archivos/ahphuelva \\
\hline Huesca & http://www.patrimonioculturaldearagon.es/archivo-historico-provincial-de-huesca \\
\hline Jaén & http://www.juntadeandalucia.es/cultura/archivos/ahpjaen \\
\hline La Rioja & https://www.larioja.org/archivo-historico/es \\
\hline Las Palmas de Gran Canaria & http://www.gobiernodecanarias.org/cultura/archivolaspalmas/ \\
\hline León & https://archivoscastillayleon.jcyl.es/web/es/nuestros-archivos/archivo-historico-provincial-leon.html \\
\hline Lleida & https://xac.gencat.cat/ca/llista_arxius_comarcals/segria/ \\
\hline Lugo & http://arquivosdegalicia.xunta.gal/portal/arquivo-historico-provincial-de-lugo/index.html \\
\hline Maó & http://www.bibliomao.es/ca/arxiu-historic \\
\hline Málaga & http://www.juntadeandalucia.es/cultura/archivos/ahpmalaga \\
\hline Melilla & $\begin{array}{l}\text { https://www.melilla.es/melillaPortal/contenedor.jsp?seccion=s_floc_d4_v1.jsp\&codbusqueda=210\&language=es\&codRe } \\
\text { si=1\&codMenuPN=601\&codMenuSN=5\&codMenu=162\&layout=contenedor.jsp }\end{array}$ \\
\hline Murcia & https://archivogeneral.carm.es/archivoGeneral/arg.inicio \\
\hline Ourense & http://arquivosdegalicia.xunta.gal/portal/arquivo-historico-provincial-de-ourense/index.html \\
\hline Palencia & https://archivoscastillayleon.jcyl.es/web/jcyl/ArchivosCastillaYLeon/es/Plantilla66y33_100/1284346754651/_/__ \\
\hline Pontevedra & http://arquivosdegalicia.xunta.gal/portal/arquivo-historico-provincial-de-pontevedra/index.html \\
\hline Protocolos de Madrid & http://www.madrid.org/archivos/index.php/quienes-somos/conocenos/archivo-historico-de-protocolos \\
\hline Real Chancillería de Granada & http://www.juntadeandalucia.es/cultura/archivos/web_es/realchancilleria \\
\hline Real y General de Navarra & http://www.navarra.es/home_es/Temas/Turismo+ocio+y+cultura/Archivos/Archivos/Archivo+General+de+Navarra/ \\
\hline Reino de Galicia & http://arquivosdegalicia.xunta.gal/portal/arquivo-do-reino-de-galicia/ \\
\hline Reino de Mallorca & http://arxiuregnemallorca.com/ \\
\hline Reino de Valencia & http://ceice.gva.es/es/web/archivo-del-reino \\
\hline Salamanca & https://archivoscastillayleon.jcyl.es/web/jcyl/ArchivosCastillaYLeon/es/Plantilla66y33_100/1284346754696/_/_ \\
\hline Santa Cruz de Tenerife & http://www.gobiernodecanarias.org/cultura/archivosantacruzdetenerife/ \\
\hline Segovia & http://archivoscastillayleon.jcyl.es/web/jcyl/ArchivosCastillaYLeon/es/Plantilla66y33_100/1284346754743/_/___ \\
\hline Sevilla & http://www.juntadeandalucia.es/cultura/archivos/ahpsevilla \\
\hline Soria & https://archivoscastillayleon.jcyl.es/web/es/nuestros-archivos/archivo-historico-provincial-soria.html \\
\hline Tarragona & https://xac.gencat.cat/ca/llista_arxius_comarcals/tarragones/ \\
\hline Teruel & http://www.patrimonioculturaldearagon.es/archivo-historico-provincial-de-teruel \\
\hline Toledo & https://cultura.castillalamancha.es/archivos/archivos-historicos-provinciales/archivo-historico-provincial-de-toledo \\
\hline Valladolid & https://archivoscastillayleon.jcyl.es/web/es/nuestros-archivos/archivo-historico-provincial-valladolid.html \\
\hline Zamora & http://www.jcyl.es/web/jcyl/Portada/es/Plantilla100Directorio/1248366924958/0/1142233579265/DirectorioPadre \\
\hline Zaragoza & http://www.patrimonioculturaldearagon.es/archivo-historico-provincial-de-zaragoza \\
\hline
\end{tabular}

